EPJ Web of Conferences 85, 02023 (2015)

DOI: $10.1051 /$ epjconf/ 20158502023

C) Owned by the authors, published by EDP Sciences, 2015

\title{
Monte Carlo Generators for Studies of the 3D Structure of the Nucleon
}

\author{
Harut Avakian ${ }^{1, a}$ \\ ${ }^{1}$ Jefferson Laboratory, 12000 Jefferson Avenue, Newport News, VA 23606, USA
}

\begin{abstract}
Extraction of transverse momentum and space distributions of partons from measurements of spin and azimuthal asymmetries requires development of a self consistent analysis framework, accounting for evolution effects, and allowing control of systematic uncertainties due to variations of input parameters and models. Development of realistic Monte-Carlo generators, accounting for TMD evolution effects, spin-orbit and quarkgluon correlations will be crucial for future studies of quark-gluon dynamics in general and 3D structure of the nucleon in particular.
\end{abstract}

\section{Introduction}

The Parton Distribution Functions (PDFs) describe the number densities of partons carrying a momentum fraction $x$ in a frame where the nucleon has very large momentum. At large values of $x$, PDFs are dominated by "valence" quarks which determine the quantum numbers of hadrons, such as electric charge and isospin. In last decades Deep Inelastic Scattering (DIS) of leptons off nucleons, emerged as a major tool to study PDFs. Quantum Chromodynamics (QCD) provides crucial guidance for the phenomenology of PDFs in the shape of factorization theorems and evolution equations describing the dependence of PDFs on the hard scale $Q$, which in DIS is associated with the fourmomentum transfer to the nucleon. However, QCD is not (yet) able to make definitive predictions of the $x$ dependence of PDFs at a given scale, which eventually have to be extracted from the data.

Standard global fitting methodologies and error analysis have been developed during decades of PDF measurements [1-4]. Recent studies have shown that especially in the region of large $x$, where little or no direct experimental information is available, the uncertainty related to the choice of parametrization and methodology may be as large or larger than the statistical uncertainty $[5,6]$, see Fig. 1. Studies by HERAPDF [5] show that already for $x>0.2$ the parametrization uncertainty for unpolarized PDFs is bigger than the statistical uncertainty. Similar conclusion was derived from studies of polarized PDFs using different input parametrizations [6].

This indicates that understanding the dynamics is of paramount importance already for studies of 1D PDFs, let alone for studies of the 3D nucleon structure. 3D information on the nucleon is encoded in transverse momentum dependent parton distributions (TMDs) and generalized parton distributions (GPDs). As a generalization of

\footnotetext{
ae-mail: avakian@jlab.org
}

PDFs, these functions have initiated a new phase in our exploration of the nucleon, and help to address long-standing questions concerning the orbital motion of quarks and gluons inside the nucleon, and their spin and spatial distributions. The recent past has witnessed enormous progress in theoretical and experimental methods for the description of these functions. In this contribution we discuss the highest-priority tasks to be addressed in the near future to advance the understanding of the 3D structure of hadrons, mainly focusing on the importance of Monte Carlo event generators capable of treating transverse and spin degrees of freedom.

\section{MC Generators}

Monte-Carlo simulations of high energy processes, so far, were focused primarily on the general features of parton showers and hadronization. There are two main classes of event generators, serving different purposes. Main applications include (1) estimating rates of certain type of events, (2) minimizing signal to background conditions, (3) planning and (4) optimizing detector performances, (5) developing analysis frameworks. The first three typically require full event generators where sets of outgoing particles are produced in the interactions between two incoming particles and a complete event is generated attempting to reproduce the raw data.

The generators developed by high energy community constructed over the past decades, including PYTHIA [10, 11], LEPTO [12], HERWIG [13] and SHERPA [14], based on a combination of analytical results and various QCDbased models, describe well most of the features visible in experiments dedicated to studies of PDFs. Spin effects due to nucleon polarization or polarized partons in the nucleon and fragmentation process are not accounted for in them, as spin in hard scattering is a very challenging concept to implement in a consistent way. As a result standard 

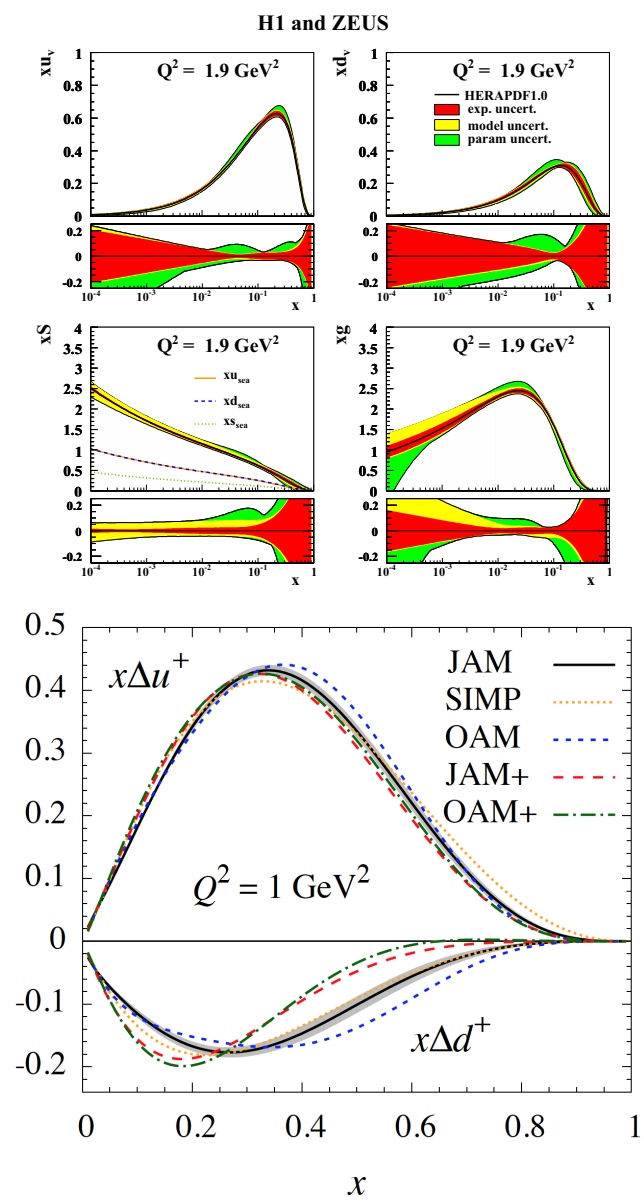

Figure 1. The unpolarized PDFs at $Q^{2}=1.9 \mathrm{GeV}^{2}$ from HERAPDF1.0 [5] (left and middle panels) and spin-dependent PDFs $\left(\Delta q^{+} \equiv \Delta q+\Delta \bar{q}\right)$ at $Q^{2}=1 \mathrm{GeV}^{2}$ [6] (right panel) from different parametrizations.

generators, which are invaluable tools for various experimental and phenomenological studies, fail to describe the new class of measurements, which focuses on azimuthal distributions of final state hadrons and requires detailed understanding of the complex dynamics of spin-orbit correlations.

Recent measurements of large spin and azimuthal asymmetries worldwide [15-19] cannot be described in terms of a 1D framework and are attributed to orbital motion of quarks, which requires an extension of PDFs to include also transverse degrees of freedom. This can be done in two complementary ways. GPDs [20-23] describe the spatial distribution of partons, which carry the longitudinal momentum fraction $x$, in the transverse ("impact parameter") plane [24]. TMDs [25-28] describe partons, which carry the longitudinal momentum fraction $x$ and transverse momentum $k_{\perp}$.

Distributions of partons in coordinate- and momentum-space as encoded in GPDs and TMDs respectively, have been widely recognized as key objectives of the JLab $12 \mathrm{GeV}$ upgrade and the polarized $p p$ program at RHIC, and are a key part of the physics program of an Electron Ion Collider. Data on spin-azimuthal distributions of hadrons in semi-inclusive DIS (SIDIS) and polarized hadron-hadron collisions, which provide access to TMDs, have been accumulated in recent years by several collaborations, including HERMES, COMPASS, BELLE, BaBar, Halls A, B, C at JLab, and PHENIX and STAR at RHIC (BNL). After the first years of running of JLab12 and the upcoming polarized pp runs at RHIC, a significantly greater set of data will be available. Although the interest in TMDs has grown enormously we are still in need of a consistent theoretical and phenomenological description spanning the full kinematic regime covered by the (un)polarized world-data. Some TMDs have been already phenomenologically extracted for $u$ and $d$ flavors by several groups, mainly from analyzing azimuthal distributions of single hadrons in SIDIS. To obtain a full picture about the 3D momentum structure of the partons in the nucleon from high to low $x$ it is important to connect the theoretical approaches to extract TMDs as well as their evolution. The studies of 3D PDFs in general, and TMDs in particular, require a lot more attention to uncertainties due to input parametrizations, as more degrees of freedom and bigger number of input parameters may generate uncontrolled model uncertainties. This makes the development of a framework for testing different extraction procedures, based on realistic Monte-Carlo generators, a high priority task for the community involved in studies of 3D PDFs.

\section{Azimuthal distributions of hadrons in electroproduction}

The calculation of any production cross sections relies upon a knowledge of the distribution of the momentum fraction $x$ and transverse momentum $p_{T}$ of the partons (quarks and gluons) in the incoming hadrons in the relevant kinematic range. These parton densities cannot be calculated perturbatively but rather are determined by global fits to data from SIDIS, Drell-Yan (DY), and, if applicable, from jet production at energy ranges of interest. In addition to having the best estimates for the values of the TMDs in a given kinematic range, it is important to understand their uncertainties. The partonic content of hadrons needs to be well understood and of sufficiently high precision if theoretical predictions are to match the experimental accuracy expected from future high precision measurements.

We consider the SIDIS process $\ell(l)+N(P) \rightarrow \ell\left(l^{\prime}\right)+$ $h\left(P_{h}\right)+X$ where $\ell$ denotes the beam lepton, $N$ the nucleon target, and $h$ the produced hadron with four-momenta given in parentheses. The four-momentum transfer is $q=l-l^{\prime}$ with $Q^{2}=-q^{2}$. Then, the relevant SIDIS variables are defined as $x=Q^{2} /(2 P \cdot q), y=(P \cdot q) /(P \cdot l)$ and $z=\left(P \cdot P_{h}\right) /(P \cdot q)$. The component of the momentum of the produced hadron transverse with respect to the virtual photon is denoted by $P_{h \perp}$ and $P_{h \perp}=\left|P_{h \perp}\right|$. Assuming single photon exchange, the lepton-hadron cross section can be expressed in a model-independent way by a set of 
structure functions, see e.g. [29], The SIDIS cross section

$$
\frac{d \sigma^{e^{-} N \rightarrow e^{-} h X}}{d x d y d \phi_{s} d z d \phi_{h} d P_{h \perp}^{2}}
$$

could be presented a set of contributions from different structure functions, depending on the beam and target spin states depending on the azimuthal angle of the produced hadron $\phi_{h}$ and the azimuthal angle of the target's polarization vector, $\phi_{S}$. The structure functions $F_{X Y Z}^{\text {weight }}$ are functions of $x, Q^{2}, z$ and $P_{h \perp}^{2}$. The subscripts $X, Y=\{U, L, T\}$ indicate the respective polarizations of beam and target, the subscript $Z=\{L, T\}$ indicates the polarization of the virtual photon where needed. The superscript "weight" reminds us of the kind of angular distribution of the produced hadrons. Furthermore, the ratio of longitudinal to transverse photon flux is $\varepsilon=\left(1-y-\frac{1}{4} \gamma^{2} y^{2}\right) /\left(1-y+\frac{1}{2} y^{2}+\right.$ $\left.\frac{1}{4} \gamma^{2} y^{2}\right)$ with $\gamma=2 M x / Q$. The polarization of the lepton is described by the helicity $\lambda_{e}$, and $\left(S_{T}, S_{L}\right)$ is the nucleon polarization vector with $S_{T}=\left|S_{T}\right|$.

Depending on kinematics, the structure functions can be interpreted in terms of transverse momentum dependent parton distribution and fragmentation functions, or in terms of the equally interesting but so far less studied fracture functions. By exploring different beam and target polarizations, and detecting a variety of final state hadrons (pions, kaons) one can access a wealth of novel information about the 3D spin structure of the nucleon, as well as spin-orbit effects in the target fragmentation region $[29,30]$.

Following the TMD factorization theorem one can write SIDIS structure functions in the current fragmentation region as a convolution of perturbatively calculable part $\mathcal{H}_{X Y}^{a}\left(Q^{2}\right)$, and universal TMD PDFs and TMD fragmentation functions [31-34]. The current knowledge of TMDs is based on theoretical studies in quark models, first lattices studies, and phenomenological extractions. First attempts to access information on Mellin moments of TMDs in Euclidean space-time lattice QCD simulations were reported [35-37]. One of the most exciting predictions from lattice studies were observations of strong spin and flavor dependences of $k_{\perp}$-distributions of partons $[36,37]$. Recently a new approach was proposed to access also the $x$-dependence of PDFs and TMDs [38, 39] from lattice QCD. More work is needed [40], but first calculations [41] indicate feasibility of the method.

Compared to PDFs, the status of TMD extractions is still in an "exploratory stage." The early efforts to extract information on the novel TMDs were largely focused on the Sivers function [42-48] and transversity [4851] using SIDIS data, and Boer-Mulders function [5256] using SIDIS and DY data. In the early studies TMD evolution was neglected or crudely estimated, which was presumably justified when working with existing SIDIS data. With the advent of convenient TMD evolution equations advances towards more quantitative treatments became possible [57-68]. While many theoretical questions remain open, further progress will be crucially driven by data, such as the recent COMPASS data [69], which were analyzed in $[70,71]$. Phenomenology efforts have been summarized recently by introduction of a library of fits and parametrizations for transverse-momentumdependent parton distribution functions (TMD PDFs) and fragmentation functions (TMD FFs) together with an online plotting tool, TMDplotter [72].

Further progress in the interpretation of the quantitative description of azimuthal, spin-orbit, and polarization phenomena in SIDIS at JLab 12 and EIC, and other processes (DY at RHIC, DY at COMPASS) will require novel $\mathrm{MC}$ technologies.

The effects involving unpolarized partons, such as Cahn and Sivers azimuthal modulations of the transverse momentum of the active quark before hard scattering and hadronization can be incorporated in generators with small modifications in existing code, as it has been done in mLEPTO [73, 74] and LEPTO-PHI [75]. The incorporation of spin effects involving the polarized parton hadronization, on the other hand, require much more efforts. Here a microscopic-level model describing the fragmentation mechanism is needed. Theoretical developments of transversely polarized quark fragmentation within the string fragmentation framework have been undertaken in [76-78], while the NJL-jet model calculations have been incorporated into a stand-alone MC generator in $[79,80]$. Thus one approach in building a fully-fledged MC event generator would be to develop the models for the hadronization of polarized quark, quark polarization transfer in each hadron emission for cascade-type mechanisms, the production and strong decays of vector mesons (this is especially important in dihadron studies), etc and test them on stand alone MC framework, such as the one developed for NJL-jet.

Both, full event generators and single-particle generators were extensively used for studies of different hard scattering processes and development of analysis frameworks.

Below is an example of studies of the effects of limited energy on the available phase space to generate high $P_{T}$ events in SIDIS. Multidimensional generator starting from initial $k_{\perp}$ of quarks and accounting for energy conservation across the production process has been used to study the final generated $k_{\perp}$ distributions in comparison with input ones. Figure 2 (right panel) shows final distributions (triangles up and down) for two different beam energies, compared to the same initial input Gaussian distribution for given fixed values of $x$ and $z$. The effect of suppression of high $P_{T}$ events due to limited phase space may have been observed by Torino group [71] in attempt to describe $P_{T}$-distributions measured at JLab Hall-C experiment [81].

\subsection{Azimuthal distributions and acceptance studies}

Any experiment has a minimum angle for detection, which introduces some $\phi$ modulation depending on the kinematical variable. An example of the CLAS12 acceptance for hadrons production in the range of $P_{T}$ from 0.4 to $0.6 \mathrm{GeV}$, defined as the ratio of reconstructed events and generated events is shown in the Fig.3. 

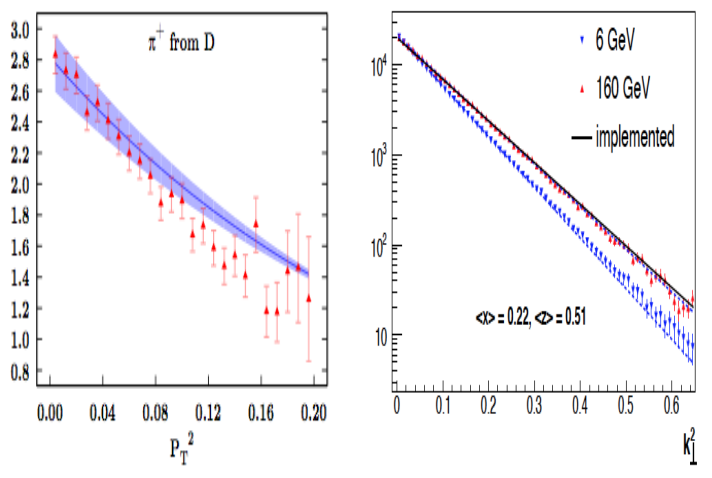

Figure 2. Calculations of Torino group [71] using simple Gaussian approximation compared to JLab Hall-C data [81](left) and MC generated distributions implementing Gaussian distribution (right). The triangles up (red) show the outcome from the $\mathrm{MC}$ at $160 \mathrm{GeV}$ initial lepton energy and triangles down (blue) show the outcome from the $\mathrm{MC}$ at $6 \mathrm{GeV}$ with dashed lines representing fits to generated distribution to a Gaussian.

In real life experiments, one measures a convolution of cross section and detector acceptance. One can describe the detector acceptance $\mathrm{A}\left(x, Q^{2}, P_{T}, z, \phi\right)$ with Fourier series, where the constants $A_{m}$ and $B_{n}$ depend on kinematical variables:

$$
A\left(x, Q^{2}, P_{T}, z, \phi\right)=A_{0}\left(1+\sum_{m=1}^{\infty} A_{m} \cos m \phi+\sum_{n=1}^{\infty} B_{n} \sin n \phi\right)
$$

With knowledge of the acceptance function provided by a realistic MC simulation one can extract azimuthal moments from complicated product using the formulae for a product of two Fourier series.
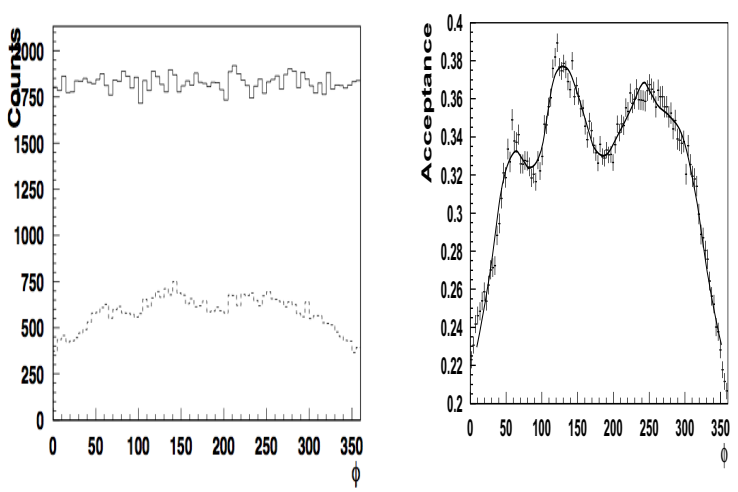

Figure 3. Generated (flat) and reconstructed $e p \rightarrow e^{\prime} \pi^{+}$events for CLAS12 detector and $11 \mathrm{GeV}$ electron beam (left panel). The right shows the acceptance (ratio of reconstructed to generated) fitted with Fourier series (first 10 cos moments and first 4 sin moments.

The acceptance has weak dependence on input cross sections, but for precision studies of azimuthal distributions this weak dependence may be crucial. In particular two initial $\phi$-distributions, $\phi$-independent and $\phi$-dependent with $\langle\cos \phi\rangle=0.15$ shown on Fig. 4, yield $\cos \phi$ moments from acceptance equal to $5.4 \%$ and $4.5 \%$, respectively. That variation may be much stronger at higher $z$ and $P_{T}$, indicating the importance of precision description of spin-azimuthal terms in hard scattering processes aiming extraction of different azimuthal moments.

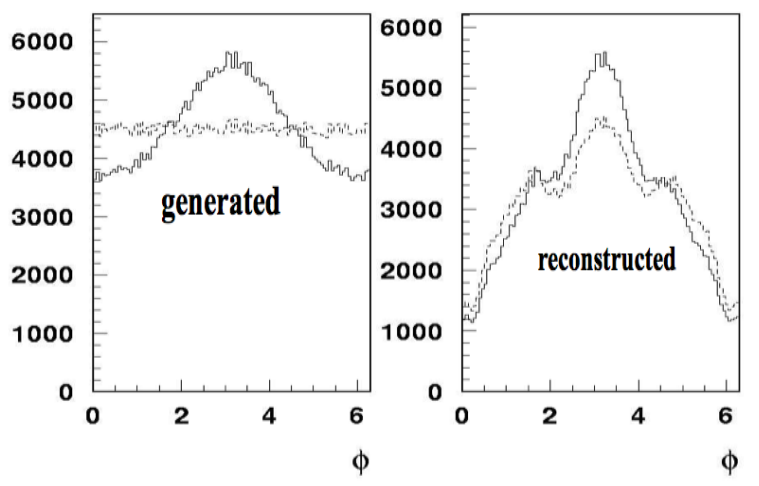

Figure 4. Two generated $\phi$-dependencies (with and without $\phi$ modulation (left) and their reconstructed versions in $e p \rightarrow e^{\prime} \pi^{+}$ events for CLAS12 detector and $11 \mathrm{GeV}$ electron beam.

The realistic MC with solid fundamentals will be important part of the global 3D analysis framework, including the data analysis, phenomenology and extended 3D PDF library (see Fig.5). These and other features of the required MC event generators were vividly discussed at the dedicated INT workshop in Seattle [82].

\section{Conclusions}

The 3D partonic structure accessible in hard scattering is rich and complex. The understanding of the contributions to final transverse momentum dependence of different azimuthal moments in the cross section [83] will require detailed studies. Monte-Carlo event generators accounting for spin-orbit correlations will be crucial to study the dependence on different model inputs, as well as sensitivity of extraction of underlying TMDs on various experimental uncertainties including acceptances, resolutions and radiative corrections. The future $3 \mathrm{D}$ nucleon structure extraction framework, should include in addition to extraction procedures a library of 3D PDFs and MC simulation frameworks using that library as input and allowing to check the quality of extracted PDFs for specific experimental conditions in a full range of accessible kinematics.

\section{Acknowledgements}

The author would like to thank the organizers for their generous support. This work was supported by DOE contract No. DEAC05-06OR23177, under which Jefferson Science Associates, LLC operates Jefferson Lab.

\section{References}

[1] A.D. Martin, R. Roberts, W. Stirling, R. Thorne, Eur.Phys.J. C23, 73 (2002), hep-ph/0110215 


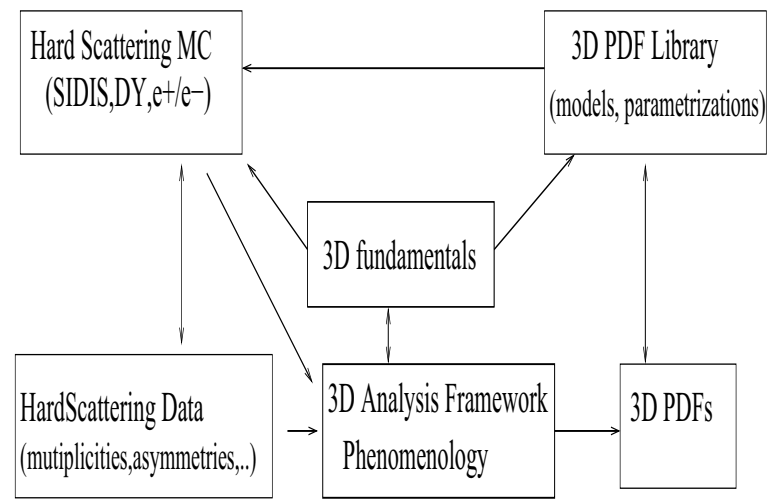

Figure 5. Global analysis framework.

[2] J. Pumplin, D. Stump, J. Huston, H. Lai, P.M. Nadolsky et al., JHEP 0207, 012 (2002), hep-ph/0201195

[3] A.D. Martin, W.J. Stirling, R.S. Thorne, G. Watt, Eur. Phys. J. C63, 189 (2009), 0901.0002

[4] H.L. Lai, M. Guzzi, J. Huston, Z. Li, P.M. Nadolsky et al., Phys.Rev. D82, 074024 (2010), 1007. 2241

[5] F. Aaron et al. (H1 and ZEUS Collaboration), JHEP 1001, 109 (2010), 0911 . 0884

[6] P. Jimenez-Delgado, H. Avakian, W. Melnitchouk (Jefferson Lab Angular Momentum (JAM) Collaboration) (2014), 1403. 3355

[7] R.D. Ball, L. Del Debbio, S. Forte, A. Guffanti, J.I. Latorre et al., Nucl.Phys. B838, 136 (2010), 1002.4407

[8] R.D. Ball et al. (NNPDF Collaboration), Nucl.Phys. B855, 153 (2012), 1107. 2652

[9] E.R. Nocera, R.D. Ball, S. Forte, G. Ridolfi, J. Rojo (The NNPDF Collaboration) (2014), 1406.5539

[10] T. Sjostrand, S. Mrenna, P.Z. Skands, JHEP 0605, 026 (2006), hep-ph/0603175

[11] T. Sjostrand, S. Mrenna, P.Z. Skands, Comput.Phys.Commun. 178, 852 (2008), 0710. 3820

[12] G. Ingelman, A. Edin, J. Rathsman, Comput.Phys.Commun. 101, 108 (1997), hep-ph/9605286

[13] G. Corcella, I. Knowles, G. Marchesini, S. Moretti, K. Odagiri et al., JHEP 0101, 010 (2001), hep-ph/0011363

[14] T. Gleisberg, S. Hoeche, F. Krauss, M. Schonherr, S. Schumann et al., JHEP 0902, 007 (2009), 0811.4622

[15] A. Bravar et al. (Fermilab E704), Phys. Rev. Lett. 77, 2626 (1996)

[16] A. Airapetian et al. (HERMES), Phys. Rev. Lett. 84, 4047 (2000), hep-ex/9910062

[17] H. Avakian et al. (CLAS), Phys. Rev. D69, 112004 (2004), hep-ex/0301005

[18] A. Airapetian et al. (HERMES), Phys. Rev. Lett. 94, 012002 (2005), hep-ex/0408013

[19] V.Y. Alexakhin et al. (COMPASS), Phys. Rev. Lett. 94, 202002 (2005), hep-ex/0503002
[20] D. Mueller, D. Robaschik, B. Geyer, F.M. Dittes, J. Horejsi, Fortschr. Phys. 42, 101 (1994), hep-ph/9812448

[21] X.D. Ji, Phys. Rev. Lett. 78, 610 (1997), hep-ph/9603249

[22] A.V. Radyushkin, Phys. Lett. B380, 417 (1996), hep-ph/9604317

[23] J.C. Collins, L. Frankfurt, M. Strikman (1997), hep-ph/9709336

[24] M. Burkardt, Int. J. Mod. Phys. A18, 173 (2003), hep-ph/0207047

[25] A. Kotzinian, Nucl. Phys. B441, 234 (1995), hep-ph/9412283

[26] P.J. Mulders, R.D. Tangerman, Nucl. Phys. B461, 197 (1996), hep-ph/9510301

[27] D. Boer, P.J. Mulders, Phys. Rev. D57, 5780 (1998), hep-ph/9711485

[28] J.C. Collins, Acta Phys.Polon. B34, 3103 (2003), hep-ph/0304122

[29] A. Bacchetta et al., JHEP 02, 093 (2007), hep-ph/0611265

[30] M. Anselmino, V. Barone, A. Kotzinian, Phys. Lett. B699, 108 (2011), 1102. 4214

[31] J.C. Collins, D.E. Soper, Nucl. Phys. B193, 381 (1981)

[32] X. Ji, J. Ma, F. Yuan, Phys. Rev. D71, 034005 (2005), hep-ph/0404183

[33] J. Collins (2011)

[34] S.M. Aybat, T.C. Rogers, Phys.Rev. D83, 114042 (2011), 1101.5057

[35] P. Hagler, B.U. Musch, J.W. Negele, A. Schafer, Europhys. Lett. 88, 61001 (2009), hep-lat/0908.1283

[36] B.U. Musch, P. Hagler, J.W. Negele, A. Schafer, Phys.Rev. D83, 094507 (2011), 1011. 1213

[37] B. Musch, P. Hagler, M. Engelhardt, J. Negele, A. Schafer, Phys.Rev. D85, 094510 (2012), 1111.4249

[38] X. Ji, Phys.Rev.Lett. 110, 262002 (2013), 1305.1539

[39] X. Ji, P. Sun, X. Xiong, F. Yuan (2014), 1405.7640

[40] Y.Q. Ma, J.W. Qiu (2014), 1404.6860

[41] H.W. Lin, J.W. Chen, S.D. Cohen, X. Ji (2014), 1402.1462

[42] A.V. Efremov, K. Goeke, S. Menzel, A. Metz, P. Schweitzer, Phys. Lett. B612, 233 (2005), hep-ph/0412353

[43] M. Anselmino, M. Boglione, J. Collins, U. D'Alesio, A. Efremov et al., pp. 236-243 (2005), hep-ph/0511017

[44] J.C. Collins et al., Phys. Rev. D73, 014021 (2006), hep-ph/0509076

[45] M. Anselmino et al., Phys. Rev. D71, 074006 (2005), hep-ph/0501196

[46] S. Arnold, A.V. Efremov, K. Goeke, M. Schlegel, P. Schweitzer, hep-ph/0805.2137 (2008), hep-ph/0805.2137 
[47] M. Anselmino, M. Boglione, U. D'Alesio, A. Kotzinian, S. Melis et al., Eur.Phys.J. A39, 89 (2009), 0805.2677

[48] W. Vogelsang, F. Yuan, Phys. Rev. D72, 054028 (2005), hep-ph/0507266

[49] A.V. Efremov, K. Goeke, P. Schweitzer, Phys. Rev. D73, 094025 (2006), hep-ph/0603054

[50] M. Anselmino, M. Boglione, U. D’Alesio, A. Kotzinian, F. Murgia et al., Phys.Rev. D75, 054032 (2007), hep-ph/0701006

[51] M. Anselmino, M. Boglione, U. D’Alesio, S. Melis, F. Murgia et al., Phys.Rev. D87, 094019 (2013), 1303.3822

[52] D. Boer, Phys.Rev. D60, 014012 (1999), hep-ph/9902255

[53] Z. Lu, B.Q. Ma, I. Schmidt, Phys.Lett. B639, 494 (2006), hep-ph/0702006

[54] B. Zhang, Z. Lu, B.Q. Ma, I. Schmidt, Phys. Rev. D77, 054011 (2008), hep-ph/0803. 1692

[55] Z. Lu, I. Schmidt, Phys. Rev. D81, 034023 (2010), 0912.2031

[56] V. Barone, S. Melis, A. Prokudin, Phys. Rev. D81, 114026 (2010), 0912 . 5194

[57] S.M. Aybat, J.C. Collins, J.W. Qiu, T.C. Rogers, Phys.Rev. D85, 034043 (2012), 1110.6428

[58] S.M. Aybat, A. Prokudin, T.C. Rogers, Phys.Rev.Lett. 108, 242003 (2012), 1112.4423

[59] J.C. Collins, T.C. Rogers, Phys.Rev. D87, 034018 (2013), 1210.2100

[60] Z.B. Kang, A. Prokudin, Phys.Rev. D85, 074008 (2012), 11 pages, 7 figures, 1201.5427

[61] Z.B. Kang, I. Vitev, H. Xing, Phys.Rev. D87, 034024 (2013), 1212.1221

[62] Z.B. Kang, B.W. Xiao, Phys.Rev. D87, 034038 (2013), 1212.4809

[63] M. Anselmino, M. Boglione, S. Melis (2012), 1204.1239

[64] A. Bacchetta, A. Prokudin, Nucl.Phys. B875, 536 (2013), 1303.2129
[65] P. Sun, F. Yuan, Phys.Rev. D88, 034016 (2013), 1304.5037

[66] P. Sun, F. Yuan, Phys.Rev. D88, 114012 (2013), 1308.5003

[67] D. Boer, Nucl.Phys. B874, 217 (2013), 1304 . 5387

[68] M.G. Echevarria, A. Idilbi, Z.B. Kang, I. Vitev, Phys.Rev. D89, 074013 (2014), 1401. 5078

[69] C. Adolph et al. (COMPASS), Eur.Phys.J. C73, 2531 (2013), 1305.7317

[70] A. Signori, A. Bacchetta, M. Radici, G. Schnell, JHEP 1311, 194 (2013), 1309. 3507

[71] M. Anselmino, M. Boglione, J. Gonzalez H., S. Melis, A. Prokudin, JHEP 1404, 005 (2014), 1312.6261

[72] F. Hautmann, H. Jung, M. Krämer, P. Mulders, E. Nocera et al. (2014), 1408. 3015

[73] A. Kotzinian, Proceedings of Transversity 2005, pp. 228-235 (2005), hep-ph/0510359

[74] A. Kotzinian (2005), hep-ph/0504081

[75] https://wiki.bnl.gov/eic/index.php/LEPTO-PHI

[76] X. Artru, J.C. Collins, Z. Phys. C69, 277 (1996), hep-ph/9504220

[77] X. Artru, arXiv:hep-ph/0207309 (2002), hep-ph/0207309

[78] X. Artru (2010), 1001. 1061

[79] H.H. Matevosyan, A.W. Thomas, W. Bentz, Phys.Rev. D86, 034025 (2012), 1205. 5813

[80] H.H. Matevosyan, A. Kotzinian, A.W. Thomas, Phys.Lett. B731, 208 (2014), 1312 . 4556

[81] R. Asaturyan, R. Ent, H. Mkrtchyan, T. Navasardyan, V. Tadevosyan et al., Phys.Rev. C85, 015202 (2012), 1103.1649

[82] INT Workshop INT-14-55W, http://www.int.washington.edu/PROGRAMS/1455w/ Organizers: Elke Aschenauer, Barbara Pasquini, Harut Avakian, Peter Schweitzer (2014)

[83] C. Aidala, B. Field, L. Gamberg, T. Rogers, Phys.Rev. D89, 094002 (2014), 1401. 2654 